\title{
EXACT CONTROLLABILITY OF THE HEAT EQUATION IN ANY DIMENSION BY ACTUATORS STRATEGIC AREAS
}

\section{CHEIKH SECK}

Laboratoire d'Analyse Numérique et d'Informatique

Université Gaston Berger

BP 234, Saint-Louis

Sénégal

Départment de Mathématiques de la FASTEF

Université Cheikh Anta Diop

Dakar

Sénégal

e-mail: cseckdiakhao@gmail.com

cheikh5.seck@ucad.edu.sn

\begin{abstract}
In this paper we show, by a new approach called SCD (surjectivity by compactness and density), the exact border controllability of the 1D heat equation by the use of strategic zone actuators.
\end{abstract}

\section{Motivations and Statement of Problem}

The exact controllability of distributed systems has in recent years a net renewed interest, thanks to the development by Lions [9, 10] of the HUM method (Hilbert Uniqueness Methods). It is essentially based on 2010 Mathematics Subject Classification: 35Q72, 93B03, 93B07, 93C20.

Keywords and phrases: control, controllability, estimations, strategic actuator.

Received August 30, 2019

(ㄷ) 2019 Scientific Advances Publishers 
the uniqueness of properties suitable for the homogeneous equation by a particular choice of controls, building a Hilbert space and a continuous linear mapping of this space into its dual Hilbert which is, in fact, an isomorphism which establishes the exact controllability.

For hyperbolic problems this method has yielded important results (Lions [9, 10], Seck et al. [3], Lebeau [7], Fursikov and Imanuvilov [6], ...); Although when the checks are supporting small (Niane [8], Jai [5]), it seems little operative, as well as for technical reasons, the multiplier method does not lead to.

As for parabolic equations any result (to our knowledge) has been prepared by this method. Also, the harmonic method is ineffective for such equations.

This work is the logical sequence of the publications (see Seck et al. $[1,2])$ where a new method has been developped to solves some of these problems types. This method is based on criteria of surjectivity of a linear continuous operator a Hilbert space in another builds directly from the exact controllability problem.

The criteria are of two types:

(1) A density criterion which is a consequence of the uniqueness properties (Lions $[9,10])$.

(2) A compactness criterion which follows from the parabolic nature of the operator or the regularity of control.

In both cases these criteria are easier to verify than HUM method of Lions.

This method which we call exact controllability by compactness and density opens up broad prospects for the theory of the exact controllability and allows for parabolic equations of Schrödinger, plates, Navier Stokes linearized solve many issues and opening up many perspectives. 


\section{Notations and Reminders}

\subsection{Functional analysis reminders}

Let $\Omega$ be a regular nonempty bounded domain of $\mathbb{R}^{N}$ of border $\Gamma$, of outer unit normal $\nu(\sigma)$ at point $\sigma \in \Gamma$.

We denote $A$ the unbounded operator of $L^{2}(\Omega)$ defined by

$$
\begin{gathered}
D(A)=\left\{u \in H_{0}^{1}(\Omega) /-\Delta u \in L^{2}(\Omega)\right\}, \\
A u=-\Delta u, \forall u \in D(A) .
\end{gathered}
$$

The operator $A$ has a Hilbert base of eigenfunctions $\left(w_{k}\right)_{k \geq 1}$ such that the sequence of associated eigenvalues $\left(\lambda_{k}\right)_{k \geq 1}$ is decreasing.

Let $T>0$, we consider the following Hilberts spaces and their respective dual. Let $\alpha \in \mathbb{R}, \beta \in\{1,0,-1\}$.

$$
F_{T}^{\alpha, \beta}=\left\{u=\sum_{k=1}^{+\infty} u_{k} w_{k} / \sum_{k=1}^{+\infty} \lambda_{k}^{\alpha} u_{k}^{2} e^{2 \lambda_{k} T \beta}<+\infty\right\} .
$$

We equip $F_{T}^{\alpha, \beta}$ with the natural scalar product

$$
(x, y)_{F_{T}^{\alpha, \beta}}=\sum_{k=1}^{+\infty} u_{k} w_{k} / \sum_{k=1}^{+\infty} \lambda_{k}^{\alpha} u_{k}^{2} e^{2 \lambda_{k} T \beta}<+\infty
$$

and, the associated norm is \|\|$_{F_{T}^{\alpha, \beta}}$.

The dual of $F_{T}^{\alpha, \beta}$ is $F_{T}^{-\alpha,-\beta}$. If $x \in F_{T}^{\alpha, \beta}$ and $y \in F_{T}^{-\alpha,-\beta}$, we have:

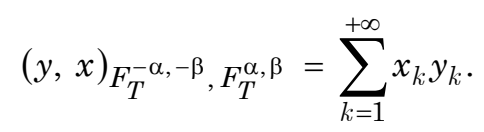


Remark 2.1. We can notice that:

(i) $F_{T}^{0,0}=L^{2}(\Omega)$,

(ii) $F_{T}^{1,0}=H_{0}^{1}(\Omega)$,

(iii) $F_{T}^{-1,0}=H^{-1}(\Omega)$.

\subsection{Case 1-D}

Let $I=] 0,1[$ an open interval of $\mathbb{R}$. We set the operator $A$ defined by

$$
D(A)=\left\{u \in H_{0}^{1}(I) /-\frac{d^{2} u}{d x^{2}} \in L^{2}(I)\right\} ; \forall u \in D(A), A u=-\frac{d^{2} u}{d x^{2}} .
$$

According to the spectral theory (Brezis [11], Hörmander [12]), $A$ admits a Hilbert base of $L^{2}(I)$ of eigenfunctions $\left(w_{k}\right)_{k \geq 1}$ whose associated eigenvalues are $\left(\lambda_{k}\right)_{k \geq 1}$ rows in the decreasing direction where

$$
\begin{cases}w_{k}(x) & =\sqrt{2} \sin k \pi x \text { and } \\ \lambda_{k} & =k^{2} \pi^{2}\end{cases}
$$

See also Lebeau [7] and Fursikov and Imanuvilov [6].

Definition 2.2. An integrable square function $\mu: I \subset \Omega \rightarrow \overline{\mathbb{R}}$ is called strategic (see Jai $[4,5]$ ) if it satisfies,

for all $\phi_{0} \in L^{2}(I)$, the solution $\phi^{+}$of heat equation

$$
\left\{\begin{array}{c}
\left.\phi^{+^{\prime}}(t, x)-\Delta \phi^{+}(t, x)=0 \text { in } Q_{T}=\right] 0,+\infty[\times I, \\
\left.\gamma \phi^{+}(t, x)=0 \text { in } \Sigma_{T}=\right] 0,+\infty[\times \partial I, \\
\phi^{+}(0)=\phi_{0} \text { in } I,
\end{array}\right.
$$

satisfies:

$$
\forall t>0, \int_{I} \mu(x) \phi^{+}(t, x) d x=0 \quad \text { then } \quad \phi_{0}=0 .
$$


Remark 2.3. (1) In 1 dimension (see Seck et al. [2]), it has been proved that there are internal strategic actuators, this is the case of certain particular geometries.

(2) Similarly, the exact controllability border of the heat equation in 1D by strategic actuators area by the SCD method (surjectivity by compactness and density) was done, see Seck et al. [1, 2].

\section{Internal Temporally Strategic Actuator}

Definition 3.1. An integrable square function

$$
\mu:\left(\begin{array}{c}
\mathbb{R}_{+}^{*} \times \Omega \rightarrow \overline{\mathbb{R}} \\
(t, x) \mapsto \mu(t, x)
\end{array}\right)
$$

is called temporally strategic if for all $\phi_{0} \in L^{2}(\Omega)$ such that

$$
\forall t \in] 0,+\infty\left[, \quad \int_{\Omega} \mu(t, x) \phi^{+}(t, x) d x=0\right.
$$

then $\phi_{0}=0$.

Remark 3.2. If the application

$$
t \mapsto \int_{\Omega} \mu(t, x) \phi^{+}(t, x) d x
$$

is analytic, it is enough that the relation is true for all $t \in] 0, T[$ so that it is true for all $t \in \mathbb{R}_{+}^{*}$.

An example of a temporally strategic actuator is given:

Theorem 3.3. If $\phi_{0} \in L^{2}(\Omega)$ and verify: $\forall k \in \mathbb{N}^{*}, \phi_{0 k} \neq 0$ for all open non-empty $\mathcal{O}$ content in $\Omega$ then $\mu(t, x)=\chi_{\mathcal{O}} \phi^{+}(t, x)$ is a temporarily strategic actuator. 
Proof 3.4. We have:

$$
\begin{aligned}
\mu_{k}(t) & =\int_{\Omega} \chi_{\mathcal{O}} \phi^{+}(t, x) w_{k}(x) d x \\
& =\int_{\mathcal{O}} \phi^{+}(t, x) w_{k}(x) d x \\
& =\sum_{h=1}^{+\infty} \phi_{0 h} e^{-\lambda_{h} t} \int_{\mathcal{O}} w_{h} w_{k} d x .
\end{aligned}
$$

Assume $\alpha_{k h}=\int_{\mathcal{O}} w_{h} w_{k} d x$, we obtain

$$
\mu_{k}(t)=\sum_{h=1}^{+\infty} \phi_{0 h} \alpha_{k h} e^{-\lambda h^{t}}
$$

Let $\zeta_{0} \in L^{2}(\Omega)$ such that:

$$
\forall t>0, \quad \int_{\Omega} \mu(t, x) \zeta^{+}(t, x) d x=0 \text { then } \sum_{h=1}^{+\infty} \zeta_{0 k} \mu_{k}(t) e^{-\lambda_{k} t}=0,
$$

let

$$
\forall t>0, \quad \sum_{k=1}^{+\infty} \sum_{h=1}^{+\infty} \zeta_{0 k} \phi_{0 h}(t) \alpha_{k h} e^{-\left(\lambda_{k}+\lambda_{h}\right) t}=0,
$$

so $\zeta_{0 k} \phi_{0 h}(t) \alpha_{k h}=0 \forall h, k$.

As $\phi_{0 h} \neq 0$, then $\forall h, \forall k \zeta_{0 k} \alpha_{k h}=0$.

Suppose it exists $k_{0} \in \mathbb{N}^{*}$ such that $\forall h \in \mathbb{N}^{*} \alpha_{h k_{0}}=0$.

Also

$$
\forall h \in \mathbb{N}^{*}, \quad \int_{\Omega} \chi_{\mathcal{O}} w_{k_{0}}(x) w_{h}(x) d x=0,
$$

so $\chi_{\mathcal{O}} w_{k_{0}}$ is identically null.

Or $w_{k}$ is a non-zero eigenfunction, this is impossible 
Corollary 3.5. For any open non-empty contained in $\Omega$, there is a temporarily strategic $\mu$ actuator whose support contained in $\mathbb{R}_{+}^{*} \times \mathcal{O}$.

Proof 3.6. Just take $\omega \subseteq \mathcal{O}, \phi_{0} \in L^{2}(\Omega)$ such that $\forall k \in \mathbb{N}, \phi_{0 k} \neq 0$ and, to pose $\mu(t, x)=\chi_{\mathcal{O}} \omega(x) \phi^{+}(t, x)$.

\section{Exact Internal Controllability of the Heat Equation}

It is recalled that the exact controllability is reflected here by this definition:

Definition 4.1 (Reminder). The $L$ operator defined under (4.3). The system defined by (4.1) will be say exactly controllable on time $T$ if and only if $L$ is surjective.

Theorem 4.2. If $\mu$ is a temporally strategic actuator, for all $y_{0} \in F_{T}^{0,-1}$, there exists $\beta \in L^{2}(] 0, T[)$ such that the solution $y$ of the equation

$$
\left\{\begin{array}{c}
\left.y^{\prime}(t, x)-\Delta y(t, x)=\beta(t) \mu(t, x) \text { in }\right] 0, T[\times \Omega \\
\gamma y=0 \text { in }] 0, T[\times \Gamma \\
y(0, x)=y_{0}(x) \text { in } \Omega
\end{array}\right.
$$

verify $y(T)=0$.

\section{Proof 4.3. First step:}

Formal construction of the $L$ operator?

We first give a notation: If $f \in L^{2}(] 0,+\infty[\times \Omega)$ and for $t$ fixed, we write $f^{+}(s, t, x)$ the solution of the direct homogeneous heat equation of the initial data $f(t, x)$. So we have

$$
f^{+}(s, t, x)=\sum_{k=1}^{+\infty} f_{k}(t) e^{-\lambda_{k} s} w_{k}(x)
$$

where $\left(f_{k}\right)_{x \geq 1}$ is given by $f(t, x)=\sum_{k=1}^{+\infty} f_{k}(t) w_{k}$. 
In the following, we will also use the functional notation $f^{+}(s, t)$. Let $\psi_{0} \in F_{T}^{0,1}$, multiply the Equation (4.1) by $\psi^{+}$and integrating by parts:

$$
\int_{\Omega} y(T) \psi(T) d x-<y_{0}, \psi_{0}{ }_{F_{T}^{0,-1}, F_{T}^{0,1}}=\int_{0}^{T} \int_{\Omega} \mu(t, x) \psi^{-}(t, x) d x d t,
$$

let

$$
\begin{aligned}
<y_{0}, \psi_{0}{ }_{F_{T}^{0,-1}, F_{T}^{0,1}} & =-\int_{0}^{T} \beta(t)<\mu^{-}(t, t), \psi_{0}{ }_{F_{T}^{0,-1}, F_{T}^{0,1}} d t \\
& =-<\int_{0}^{T} \beta(t) \mu^{-}(t, t) d t, \psi_{0}{ }_{F_{T}^{0,-1}, F_{T}^{0,1}},
\end{aligned}
$$

from where

$$
y_{0}=-\int_{0}^{T} \beta(t) \mu^{-}(t, t) d t
$$

so

$$
L_{1}(\beta)=-\int_{0}^{T} \beta(t) \mu^{-}(t, t) d t
$$

We are posing now: $L(\beta)=-\left(L_{1}(\beta)\right)^{+}(T)$ so

$$
L(\beta)=-\int_{0}^{T} \beta(t) \mu^{+}(T-t, t) d t .
$$

\section{Second step:}

$L \in \mathcal{L}\left(L^{2}(] 0, T[), L^{2}(\Omega) ?\right.$ 
Let $\beta \in L^{2}(] 0, T[)$, we have

$$
\begin{aligned}
\sum_{k=1}^{+\infty}\left(\int_{0}^{T} \beta(t) \mu_{k}(t) e^{-\lambda_{k}(T-t)} d t\right)^{2} & \leq \sum_{k=1}^{+\infty}\|\beta\|_{L^{2}(] 0, T[)} \int_{0}^{T} \mu_{k}^{2}(t) e^{-\lambda_{k}(T-t)} d t \\
& \leq \sum_{k=1}^{+\infty}\|\beta\|_{L^{2}(] 0, T[)}\left\|\mu_{k}\right\|_{L^{2}(] 0, T[)}^{2} \\
& \leq\|\beta\|_{L^{2}(] 0, T[)}\|\mu\|_{L^{2}(] 0, T[\times \Omega)}^{2} \\
L(\beta) \in L^{2}(\Omega) \text { and } &
\end{aligned}
$$

$$
\|L(\beta)\| \leq\|\beta\|_{L^{2}(] 0, T[)}\|\mu\|_{L^{2}(] 0, T[\times \Omega)}^{2} .
$$

\section{Third step:}

$L\left(L^{2}(] 0, T[)\right)$ is dense in $L^{2}(\Omega) ?$.

Let $\psi_{0} \in F_{T}$ such that $\psi^{-}(T)$ is orthogonal to $L\left(L^{2}(] 0, T[)\right)$; let $\beta \in L^{2}(] 0, T[)$, we have

$$
\left(L(\beta), \psi^{-}(T)\right)_{L^{2}(\Omega)}=0
$$

so

$$
\begin{gathered}
\int_{\Omega} \int_{0}^{T} \beta(t) \mu^{+}(T-t, t) \psi^{-}(T) d x d t=0 \Rightarrow \\
\int_{0}^{T} \beta(t) \int_{\Omega} \mu^{+}(T-t, t) \psi^{-}(T) d x d t=0 \Rightarrow \\
\int_{0}^{T} \beta(t) \int_{\Omega} \mu(t, x) \psi^{-}(t) d x d t=0
\end{gathered}
$$


from where

$$
\forall t \in] 0, T\left[, \int_{\Omega} \mu(t, x) d t \psi^{-}(t) d x=0\right.
$$

so $\psi_{0} \equiv 0$ from where $\psi_{0}(T)=0$.

\section{Fourth step:}

$L^{*} L$ is compact of $L^{2}(] 0, T[)$ in $L^{2}(] 0, T[) ?$

$$
\begin{aligned}
(L(\beta), L(\gamma))_{L^{2}(\Omega)} & =\int_{\Omega} \int_{0}^{T} \beta(t) \mu^{+}(T-t, t) d t \int_{0}^{T} \gamma(s) \mu^{+}(T-s) d s d x \\
& =\int_{0}^{T} \int_{0}^{T} \beta(s) \gamma(t) \int_{\Omega} \mu^{+}(T-t, t) d x d s d t \\
& =\int_{0}^{T} \gamma(t) \int_{0}^{T} \beta(s)\left(\int_{\Omega} \mu^{+}(T-s, s) d x\right) d s d t,
\end{aligned}
$$

so

$$
\begin{aligned}
L^{*} L(\beta)(t) & =\int_{0}^{T} \beta(s) \int_{\Omega} \mu^{+}(T-s, s) \mu^{+}(T-t, t) d x d s \\
& =\int_{0}^{T} \beta(s) \sum_{j=1}^{+\infty} \mu_{k}(t) \mu_{k}(s) e^{-\lambda_{k}(T-s)} e^{-\lambda_{k}(T-t)} d x d s \\
& =\int_{0}^{T} \beta(s) K(t, s) d s,
\end{aligned}
$$

where

$$
K(t, s)=\sum_{j=1}^{+\infty} \mu_{k}(t) \mu_{k}(s) e^{-\lambda_{k}(T-s)} e^{-\lambda_{k}(T-t)}
$$

$K$ is continuous on $[0, T] \times[0, T]$, just take $\mu(t)$ regular and the choice of $\phi_{0}$ regulates the problem. 
Fifth step: Conclusion

Let $y_{0} \in F_{T}^{0,-1}$ then it exists $\beta \in L^{2}(] 0, T[)$ such that

$$
y^{+}(T)=-\int_{0}^{T} \beta(t) \mu^{+}(T-t, t) d t
$$

Let $\psi_{0} \in F_{T}^{0,1}$, we have

$$
\begin{aligned}
\left\langle y^{+}(T), \psi^{-}(T)\right\rangle_{L^{2}(\Omega)} & =\left\langle y_{0}, \psi_{0}\right\rangle_{F_{T}^{0,-1}, F_{T}^{0,1}} \\
& =-\int_{\Omega}\left(\int_{0}^{T} \mu^{+}(T-t, t) d t\right) \psi^{-}(T) d x \\
& =-\int_{0}^{T} \int_{\Omega} \mu(t) d t \psi^{-}(t) d x d t .
\end{aligned}
$$

On the other hand, there is the solution of

$$
\left\{\begin{array}{c}
\left.y^{\prime}-\Delta y=\beta(t) \mu(t) \text { in }\right] 0, T[\times \Omega, \\
\gamma y=0 \text { in }] 0, T[\times \Gamma, \\
y(0)=y_{0} \text { in } \Omega
\end{array}\right.
$$

then

$$
\int_{\Omega} y(T) \psi^{-}(T) d x-\left\langle y_{0}, \psi_{0}\right\rangle_{F_{T}^{0,-1}, F_{T}^{0,1}}=\int_{0}^{T} \int_{\Omega} \mu(t) \beta^{-}(t) d x d t
$$

so

$$
\int_{\Omega} y(T) \psi^{-}(T) d x=0
$$

from where $y(T)=0$. 
Remark 4.4. We can choose the control as regular as we want.

Theorem 4.5. Let $\mathcal{O}$ an open part of $\Omega$ not empty, for all $y_{0} \in F_{T}^{0,-1}$, it exists $\mu \in L^{2}(] 0, T[\times \Omega)$ to support contained in ] $0, T[\times \mathcal{O}$ such that if $y$ is solution

$$
\left\{\begin{array}{c}
\left.y^{\prime}-\Delta y=\beta(t) \mu(t) \text { in }\right] 0, T[\times \Omega, \\
\gamma y=0 \text { in }] 0, T[\times \Gamma, \\
y(0)=y_{0} \text { in } \Omega,
\end{array}\right.
$$

then $y(T)=0$.

Proof 4.6. Take $\eta$ functions to support contained in $\mathcal{O}$, belonging to $D(\Omega)$ non-zero on an open content in $\mathcal{O}$.

Let $\psi_{0} \in D(A)$ such that $\forall k \in \mathbb{N}^{*}, \phi_{0 k} \neq 0$ then $\mu(t)=\eta \phi^{+}(t)$ is temporally strategic and we apply the Theorem 4.3.

\section{References}

[1] C. Seck, Exact controllability border of heat equation in 1D by strategic actuator area by the SCD Method 2019, Soumis

[2] C. Seck, A. Sène and M. T. Niane, Exact controllability of heat equation in 1D by a strategic zone actuator 2019, Soumis

[3] C. Seck, G. Bayili, A. Séne and M. T. Niane, Contrôlabilité exacte de l'équation des ondes dans des espaces de Sobolev non réguliers pour un ouvert polygonal, Afrika Matematika 23(1) (2012), 1-9.

DOI: https://doi.org/10.1007/s13370-011-0001-6

[4] A. El Jai, Analyse régionale des systèmes distribués, ESAIM: Control, Optimisation and Calculus of Variation 8 (2002), 663-692.

DOI: https://doi.org/10.1051/cocv:2002054

[5] A. El Jai, Quelques problèmes de contrôle propres aux systèms distribués, Annals of University of Craiova: Mathematics and Computer Science Series 30 (2003), 137-153.

[6] A. V. Fursikov and O. Yu Imanuvilov, Controllability of Evolution Equations, Séoul National University, 1996. 
[7] G. Lebeau and L. Robbiano, Contrôle Exact de l'Équation de la Chaleur, Prépublications, University Paris-Sud, 1994.

[8] M. T. Niane, Contrôlabilité exacte spectrale élargie des systèmes distribués par action sur une partie analytique arbitraire de la fontière, Comptes Rendus de l'Académie des Sciences Paris, t. 309, Sèrie 1 (1989), 335-340.

[9] J.-L. Lions, Contrôlabilité exacte perturbations et stabilisation de systèmes distributes, Tome 2, Recherches en Mathématiques Appliquées, Volume 9, Perturbations, Masson, Paris, 1988.

[10] J.-L. Lions, Contrôlabilité exacte perturbations et stabilisation de systemès distributes, Tome 1, Recherches en Mathématiques Appliquées, Volume 8, Paris, 1988.

[11] Ham Brezis, Analyse Fonctionnelle: Théorie et Applications, Masson, 1983.

[12] Lars Hörmander, Linear Partial Differential Operators, Springer Verlag, Berlin, 1976, vii + 285, $35-\mathrm{XX}(46 \mathrm{FXX}), 0404822(53 \# 8622)$. 